\title{
Propagation d'ondes acoustiques dans des empilements faiblement désordonnés
}

\author{
C. CONSTANTOPOULOS et B. PERRIN
} Département de Recherches Physiques, Université P\& M Curie, T22, Boîte 136, 4 place Jussieu,
75252 Paris cedex 05, France

\begin{abstract}
This work is devoted to a numerical study of transverse waves localization propagating along the stacking axis of random alternating $\mathrm{Fe}-\mathrm{Cu}$ layers. The randomness is introduced through fluctuations of the layer thickness. For a weak disorder, the calculated localization length is in good agreement with the theoretical (asymptotic) predictions as far as a large number of plies (5000) is considered. For a more realistic number of plies, from an experimental point of view $(200<\mathrm{N}<500)$, large discrepancies appear due to the weak acoustic contrast at the interface $\mathrm{Cu}-\mathrm{Fe}$.
\end{abstract}

\section{INTRODUCTION}

Ce travail est la première étape d'une étude, théorique et expérimentale, de la propagation nonlinéaire d'ondes acoustiques dans des empilements alternés de couches métalliques d'impédances acoustiques peu différentes et d'épaisseurs aléatoires. A faible puissance acoustique (régime linéaire), ce type de structure unidimensionnelle et désordonnée, qui constitue un système modèle pour étudier la localisation d'Anderson, est particulièrement simple à mettre en oeuvre et a été étudié à plusieurs reprises [1],[2] par le formalisme de la matrice de transfert très adapté aux problèmes linéaires $1 \mathrm{D}$ [3].

Dans les milieux parfaitement périodiques, les ondes sont évanescentes pour des intervalles finis de fréquence définissant les bandes d'arrêt [4]. Le désordre induit par la variation aléatoire de l'épaisseur provoque, à toute fréquence, des interférences destructives et les ondes sont, dans la limite asymptotique des grands empilements, exponentiellement atténuées; le coefficient de cette décroissance exponentielle, par pli, définit la constante de localisation, au sens d'Anderson [5],

$$
\gamma=\lim _{N \rightarrow \infty} \frac{1}{N} \ln \left[\left|\operatorname{Tr}\left(\prod_{n=1}^{N} \tau_{n}\right)\right|\right],
$$

$\tau_{\mathrm{n}}$ étant la matrice de transfert du pli $\mathrm{n}$ [4]. A faible désordre, pour de petites fluctuations des variables aléatoires, la constante de localisation est proportionnelle au carré de la fréquence, ce qui, en dimension 1 , correspond au régime de diffusion Rayleigh sur les défauts structuraux induits par la variation aléatoire d'épaisseur. Toutefois ce résultat n'est obtenu que dans la limite asymptotique $N \rightarrow \infty$.

Nous présentons, dans cet article, les résultats d'une étude numérique relative à des ondes transversales se propageant linéairement dans un empilement alterné fer-cuivre de couches d'épaisseur moyenne $45 \mu \mathrm{m}$ qui, pour un empilement strictement périodique, conduisent à un premier gap en fréquence s'étendant de 13.36 à $15.28 \mathrm{MHz}$. Nous avons de plus choisi des tailles d'empilements réalisables au plan expérimental $(\mathrm{N} \leq 500)$. 


\section{PRORAGATION DANS LES SYSTEMES ALTERNES UNIDIMENSIONNELS}

On considère une onde élastique plane de pulsation $\omega$ traversant une séquence alternée unidimensionnelle, constituée de deux matériaux $(i=1,2)$ d'impédances acoustiques différentes $z_{i}$. Pour chaque pli $i$, on note $u_{L(R), i}$ le déplacement et $\sigma_{L(R), i}$ la contrainte normale à l'interface gauche(droite). Les grandeurs $\left(u_{R, i}, \sigma_{R, i}\right)$ s'expriment en fonction de $\left(u_{L, i}, \sigma_{L, i}\right)$ à l'aide d'une matrice de transfert unimodulaire $\tau_{i}$

$$
\tau_{i}=\left(\begin{array}{cc}
\cos k_{i} d & \frac{\sin k_{i} d}{\omega z_{i}} \\
-\omega z_{i} \sin k_{i} d & \cos k_{i} d
\end{array}\right),
$$

$\mathrm{k}_{\mathrm{i}}$ étant le vecteur d'onde à l'intérieur du pli et $\mathrm{d}$ son épaisseur.

Si l'on ne considère que des plis de même épaisseur $\mathrm{d}_{0}$, on obtient un système périodique et la matrice de transfert $\tau_{1,2}$ décrivant le passage de l'onde à travers une paire de plis a une demi-trace $t_{1,2}$ donnée par

$$
t_{1,2}=\cos k_{1} d_{0} \cos k_{2} d_{0}-r \sin k_{1} d_{0} \sin k_{2} d_{0},
$$

où $\mathrm{r}=\frac{1}{2}\left(\frac{\mathrm{z}_{1}}{\mathrm{z}_{2}}+\frac{\mathrm{z}_{2}}{\mathrm{z}_{1}}\right)$. La relation de dispersion décrivant la propagation de l'onde au travers d'un empilement périodique s'écrit alors : $\cos \left(2 k d_{0}\right)=t_{1,2}$.

Pour $\left|t_{1,2}\right| \leq 1$, le vecteur d'onde $\mathrm{k}$ est réel ; l'intervalle de fréquence respectant cette condition définit les bandes passantes. Pour $\left|t_{1,2}\right|>1$, l'onde est évanescente et sa décroissance exponentielle définit une longueur de localisation.

On considère maintenant la même séquence alternée formée de couches d'épaisseur variable $d=d_{0}+\delta$. Les phases spatiales $\alpha_{\mathrm{i}}=\mathrm{k}_{\mathrm{i}} \mathrm{d}$ définissent des variables aléatoires conduisant à des matrices de transfert $\tau\left(\alpha_{i}\right)$ aléatoires. Les produits infinis de matrices aléatoires $2 \times 2$ ont été largement étudiés [6] et il est, notamment, rigoureusement démontré que, pour des matrices unimodulaires, la limite définie dans l'éq. 1 existe. Calculer cette limite revient à étudier la trajectoire d'un point $\rho \mathrm{e}^{\mathrm{i} \theta} \mathrm{du}$ plan complexe sous l'action successive des matrices $\tau\left(\alpha_{i}\right): \rho_{N} e^{i \theta_{N}}=\tau(\alpha)\left(\rho_{N-1} e^{i \theta_{N-1}}\right)$. Les variables de phase $\theta_{N}$ et $\theta_{N-1}$ sont reliées par une relation de récurrence et possèdent une densité de probabilité $v(\theta)$ invariante par cette transformation. Ceci conduit à l'équation self-consistent de Dyson-Schmidt [7]

$$
v(\theta)=\int v(\theta(\alpha)) \frac{d \theta(\alpha)}{d \theta} p(\alpha) d \alpha
$$

où $\mathrm{p}(\alpha)$ est la densité de probabilité de la variable de phase $\alpha$. Cette équation permet théoriquement de déterminer la densité $v(\theta)$ mais n'est pratiquement utilisable que dans certaines circonstances. La constante de localisation définie par l'éq. 1 se déduit alors de la densitév $(\theta)$ à l'aide de la relation

$$
\gamma=\int \ln \left|\frac{\tau(\alpha)\left(\rho \mathrm{e}^{\mathrm{i} \theta}\right)}{\rho}\right| \mathrm{p}(\alpha) v(\theta) \mathrm{d} \alpha \mathrm{d} \theta
$$

Par un développement limité en faible désordre des éqs. 4 et 5 , Baluni et al. [1] ont obtenu, pour $\mathrm{t}_{1,2}^{2}<1$, une expression de la constante de localisation (atténuation par pli) 


$$
\gamma(\omega)=\frac{\left(r^{2}-1\right) \omega^{2} \bar{\delta}^{2}\left[\left(\sin \left(k_{1} d\right) / c_{2}\right)^{2}+\left(\sin \left(k_{2} d\right) / c_{1}\right)^{2}\right]}{2\left(1-t_{12}^{2}\right)}
$$

où $c_{i}$ représente la vitesse des ondes transverses dans le pli $\mathrm{i}$ et $\bar{\delta}$ est la variance de la fluctuation d'épaisseur $\delta$. Pour les basses fréquences $\left(\mathrm{k}_{\mathrm{i}} \mathrm{d}<<1\right), \gamma(\omega)$ est proportionnel à $\omega^{1+1}$, ce qui correspond au régime de diffusion Rayleigh sur les interfaces.

\section{RESULTATS NUMERIQUES}

Nous avons choisi le couple $\mathrm{Fe}-\mathrm{Cu}$, qui présente un faible contraste acoustique $\mathrm{z}_{\mathrm{Fe}} / \mathrm{z}_{\mathrm{Cu}}=1.24$, afin d'étudier ultérieurement la propagation nonlinéaire d'ondes en milieu faiblement désordonné. Nous avons calculé numériquement la constante de localisation en moyennant le logarithme du coefficient de transmission d'un empilement de $\mathrm{N}$ plis sur quelques centaines de réalisations. On peut observer sur la fig. I que le comportement asymptotique, prédit par la relation 6, est parfaitement vérifié sur un large domaine spectral $\left(k_{i} \bar{\delta}<1\right)$ pour un nombre de plis important $(\mathrm{N}=5000)$. Il n'est toutefois pas possible de retrouver ce résultat, principalement dans le régime de diffusion Rayleigh, pour un nombre de plis réaliste sur le plan expérimental $(200<\mathrm{N}<500)$. Il apparaît en effet, sur la fig. II, que même après des moyennes sur 1000 réalisations il y a persistence d'intervalles de fréquence pour lesquels une onde acoustique peut se propager

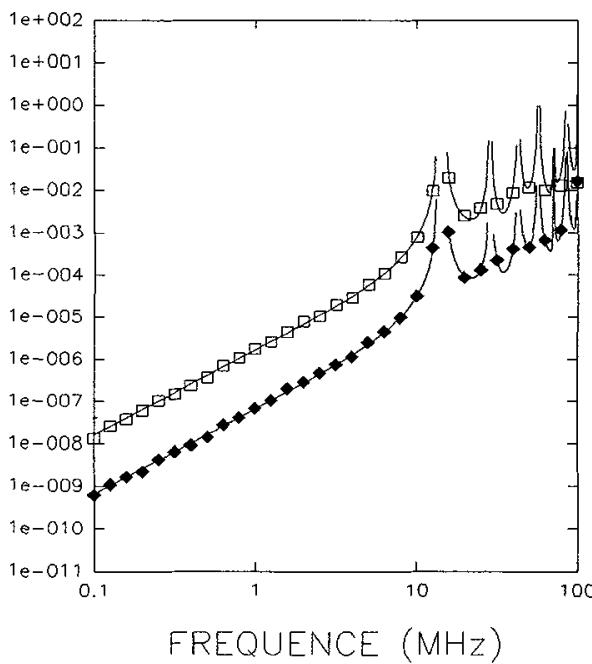

Fig I - Constante de localisation, pour un empilement de couches $\mathrm{Fe}-\mathrm{Cu}$, d'épaisseur moyenne $45 \mu \mathrm{m}$ et de variance $I(\square)$ et $5 \mu \mathrm{m}(\bullet)$, déduite du coefficient de transmission de5000 plis moyenné sur 200 réalisations. Les lignes continues représentent le comportement asymptotique en régime de faible désordre (Eq.6).

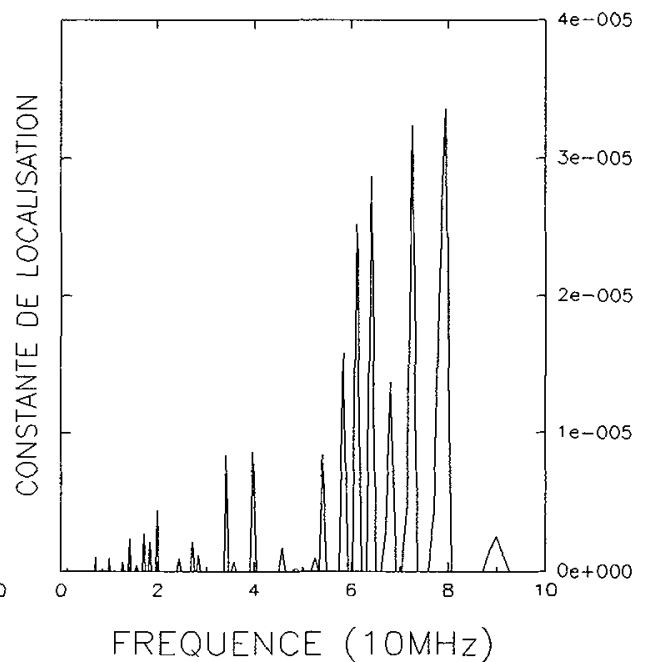

Fig II - Constante de localisation obtenue pour un empilement de 200 plis, un moyennage sur 1000 réalisations et une variance de I $\mathrm{um}$.

dans ce système désordonné. L'origine, non statistique, de ces écarts au comportement asymptotique réside dans le faible contraste acoustique du couple Fe-Cu. Dans l'empilement périodique alterné, le premier gap 
en fréquence s'ouvre à $13.36 \mathrm{MHz}$ pour une épaisseur moyenne $\mathrm{d}_{0}$ de $45 \mu \mathrm{m}$. Si l'on considère un motif élémentaire formé de plusieurs plis d'épaisseur légèrement différentes, on ouvre des gaps secondaires à des fréquences inférieures à $13.36 \mathrm{MHz}$. Le contraste acoustique et la variance des épaisseurs étant faibles, ces gaps sont très étroits et occupent une position dépendant peu de la configuration obtenue par tirage au sort. Après moyennage sur de nombreuses configurations il subsiste donc des intervalles libres entre les gaps. Lorsque l'on augmente le nombre de plis formant le motif, ces gaps commencent à se connecter; il y a toutefois rémanence de la structure ordonnée dans le spectre de transmission pour des empilements de quelques centaines de plis et des fréquences de quelques MHz. Ceci peut être observé sur la fig. III qui représente, pour une fréquence de $5 \mathrm{MHz}$, l'évolution de l'inverse du coefficient du transmission moyenné en fonction du nombre de plis traversé.

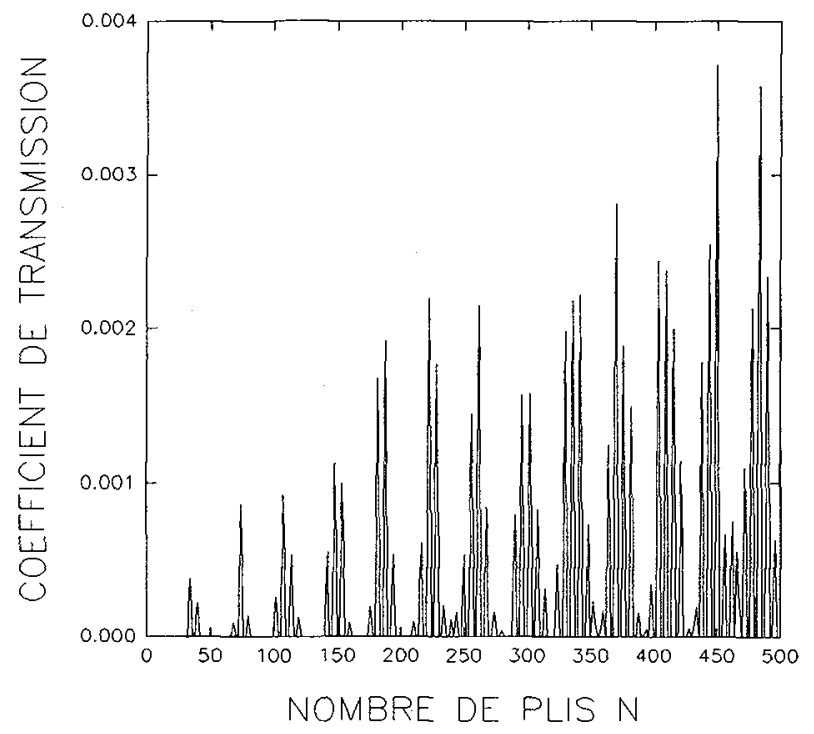

Fig III - Variation de l'inverse du coefficient de transmission en fonction du nombre de plis de l'empilement pour une fréquence de 5 $M H z$ et une variance de la fluctuation d'épaisseur de 1 pm.

Cette étude a été menée en négligeant l'absorption intrinsèque des matériaux. On peut prévoir que la prise en compte d'un coefficient d'atténuation peut amoindrir, dans une certaine mesure, les effets de rémanence de la structure périodique moyenne en fusionnant de nombreux gaps secondaires.

\section{Références}

[1] Baluni V. and Willemsen J., Phys. Rev. A 31, (1985) 3358-3363.

[2] Depollier C., Kergomard J., Lesueur J.C. et Laloé F., Revue du Cethedec-Ondes et Signal 79 (1984) 67-81.

[3] Luck J.-M., Systèmes désordonnés unidimensionnels, (Collection Aléa Saclay, 1992) p18.

[4] Brillouin L. et Parodi M., Propagation des ondes dans les milieux périodiques ( Masson-Dunod éditeurs, 1956).

[5] Anderson P.W., Phys. Rev. 109, 1492-1505 (1958).

[6] Carmona R., Lacroix J., Spectral Theory of Random Schrödinger Operators (Birkhäuser, Boston, 1990).

[7] Ziman J.M., Models of Disorder (Cambridge University Press, Cambridge, 1979). 